\title{
Modeling spatial and temporal transmission of foot-and-mouth disease in France: identification of high-risk areas
}

\author{
Arnaud Le MENACH*, Judith LEGRAND, Rebecca F. GRAIS, \\ Cécile Viboud, Alain-Jacques VAllERON, Antoine FlaHAUlT
}

INSERM Unité 444, Université Pierre et Marie Curie, Épidémiologie et Sciences de l'Information Biomédicale, 27 rue Chaligny, 75012 Paris, France

(Received 6 April 2004; accepted 10 February 2005)

\begin{abstract}
Foot-and-mouth disease is one of the most contagious diseases of animal livestock. We used statistical tools to explore the dynamics of epidemics and to evaluate the consequences of virus reintroduction in France. We developed a stochastic farm-based model adapted to the French farm structure from previous modeling works following the 2001 epidemic in the United Kingdom. This model depends upon the distance between the 280000 French farms and on species type (e.g. cows and sheep) and it tracks each animal's farm status at any given day. Since data were only available at the town scale, the farm location and the number of animals in each farm were simulated over the surface area of each French town, as well as the number of mixed farms. Based on 200 simulations of the model, our results allowed for the study of local disease transmission, since it begins simulations once limitation of movement is put into place. On average, the same 50 randomly chosen initially infected farms would lead to 1110 infected farms $(610 ; 1590)$ when two control strategies (culling within $0.5 \mathrm{~km}$ from an infected farm and vaccination within $3 \mathrm{~km}$ ) are put into place. Regions with high densities of cows and sheep (e.g. Pays-de-la-Loire) are high-risk zones, confirming that the epidemic process depends upon the location and the type of initially infected farms (size, species type). The results of this model highlight the importance of Geographical Information Systems (GIS) to obtain more precise data concerning herds.
\end{abstract}

foot-and-mouth disease / France / spatial heterogeneity / high risk zone / simulation

\section{INTRODUCTION}

Foot and mouth disease (FMD) is a highly contagious disease with economic, social (concerning human movement restriction) and medical (animal death) consequences [26]. The disease is caused by a virus belonging to the family Picornaviridae, a member of the Aphthovirus genus. The virus is highly resistant in the environment, can persist outside the host or in animal products for over one month and may be dispersed by wind over long distances [14]. It can be transmitted between herds by commercial movements, either by direct or indirect contact [28]. Infected animals excrete the virus before the end of the incubation period until recovery and the virus is mainly

\footnotetext{
* Corresponding author: arnaud.lemenach@u707.jussieu.fr
} 
found in the air or other animal products ${ }^{1}$. The disease affects sheep, cattle, goats, pigs and all wild cloven-hoofed mammals. The incubation period lasts from 3 to 11 days including a $1-4$ day presymptomatic period during which the animals are already infectious [15]. The animals then show signs of the disease characterized by fever and anorexia and a decrease in milk yield for 2 to 3 days before exhibiting acute signs. Mortality rate is very low, being at about $2 \%$, except among the young, compared to the high morbidity rate ranging from $65 \%$ to $70 \%{ }^{1}$; most animals recover from infection with permanently reduced weight gain. There are species heterogeneities in aerosol excretion and clinical signs: sheep are less susceptible than cattle but excrete the same quantities of virus and exhibit less characteristic signs. As a result, sheep may be responsible for long-range disease spread through commercial movements. Pigs excrete the virus in large quantities but are much less susceptible than sheep or cows [17]. Pigs were the index case in the 2001 outbreaks in the UK but did not play an important role in the spread of the epidemic once the disease was imported [12] and only $1 \%$ of the infected farms owned pigs. The same situation was observed in the Netherlands [6].

According to French legislation, when a herd is infected, two buffers are put into place: a $3 \mathrm{~km}$ protection buffer and a $10 \mathrm{~km}$ surveillance buffer. The infected herd is culled and other strategies, such as preemptive culling or ring vaccination may be used in these buffers [22]. From the 1960's to 1991 , annual vaccination of cows occurred as a control strategy until European law prohibited the use of vaccination against FMD. Therefore we may consider the French domestic animal population as entirely susceptible for this study.

${ }^{1}$ Office International des epizooties (OIE), Technical disease card: foot-and-mouth disease http:// www.oie.int/eng/maladies/fiches/a-A010.htm [consulted 24 January 2003].
The majority of work on the mathematical modeling of FMD addresses the 19671968 and 2001 UK epidemics. The first studies used a susceptible-exposed-infectious-removed (SEIR) [2] model of infection dynamics within the animal population. No spatial or species heterogeneity were taken into account [14]. Nevertheless, exposure to a virus has a spatial component that can influence the spatial spread of the disease: the animals are grouped by farms, farms are heterogeneously distributed among the landscape and contacts are more frequent with neighbors. The importance of spatial clustering has been emphasized for several diseases including FMD [13]. Following the 2001 UK epidemic, Ferguson et al. [9] developed a deterministic model based on a set of differential equations taking into account the spatial network of farms. No species heterogeneity has been implemented but spatial heterogeneity was taken into account. This model incorporates the effect of national movement restriction into disease spread and concluded that the $\mathrm{R}_{0}$ (i.e. the expected number of secondary infected farms from a single infected farm introduced into a totally susceptible population) decreased from 4.5 to 1.6 in regions affected by the disease after movement restriction was implemented. Durand and Mahul [8] developed a deterministic state-transition model derived from a Markov chain, where the unit of concern is an average composite herd and the time step is half a week. The model was used to compare the development of FMD epidemics in two very different regions in France and for control strategies implemented by the animalhealth authorities. Keeling et al. [18, 19] developed a stochastic model based on all British farms. Species (cows and sheep) and spatial heterogeneity were taken into account.

The goal of this research was to explore the impact of FMD reintroduction into a given area focusing on direct spread from farm to farm. In order to accomplish this, we developed a farm-based mathematical model applying the Keeling et al. method [19] to the French farm structure. We 


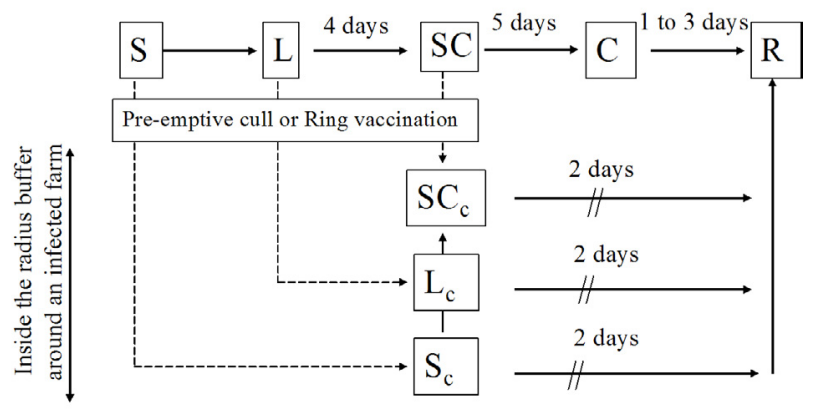

Figure 1. Modeling the infection process. Farms pass from Susceptible (S) Latent (L) depending upon the probability of infection $(\mathrm{Pe})$. Farms then become Subclinical (SC) within 4 days and the progress to Clinical (C) within 5 days. Finally they are classified as Removed (R) after the animals have been culled. If the farms are located within a certain radius containing an infected farm (C), the susceptible farms are classified as the Susceptible control $\left(\mathrm{S}_{\mathrm{c}}\right)$, the Latent one as the Latent control $\left(\mathrm{L}_{\mathrm{c}}\right)$ and the Subclinical one as the Subclinical control $\left(\mathrm{SC}_{\mathrm{c}}\right)$. They are all classified as Removed within 2 days.

focused on direct spread since national limitation of movement is put into place as soon as the disease is observed by the veterinary services and the long range transmission dramatically decreases. This model is a useful tool to evaluate the dynamics of epidemics and the impact of various control strategies on a national scale.

\section{MATERIALS AND METHODS}

\subsection{Model}

Our model structure was similar to that of Keeling et al. [19]: it depended upon spatial and species (e.g. cows and sheep) heterogeneity. We built a stochastic SEIR model at the farm level with a 1 day time step. It was reasonable to treat the farm as an individual unit because of the rapid transmission of the virus between the animals. This assumption has been used successfully in past research on rapidly transmissible diseases more especially since animals would be kept within holdings if an epidemic occurred [9, 11, 18]. First, each farm was classified as susceptible (S). When the animals of the farm became infected, the farm was classified as Latent (L). This period lasted for 4 days and then the farm was classified as Subclinical (SC) for 5 days [18]. This means that the animals excreted the virus but exhibited no signs or only prodromal signs not detected by the authorities. Then the animals exhibited acute symptoms and the farm was classified as symptomatic infectious (C). The animals were culled (R) in a delay varying from 1 to 3 days according to the 2001 UK outbreak data [18]. Thus the infectious period was assumed to be 8 days at the beginning of the epidemic and 6 days at the end (Fig. 1). We employed the mean values for disease states, since it has been previously shown that ignoring the distributed nature of the true lags did not significantly impact on either the spatial or temporal pattern of the epidemic [19].

The probability that a susceptible farm $(i)$ is infected by the neighboring infectious farms $(j)$ was:

$$
\begin{aligned}
P e_{i}= & 1-\exp \left\{-\sum_{k}\left(S u_{k} \times N_{i, k}\right):\right. \\
& \left.\times \sum_{k}\left(\operatorname{Tr}_{k} \sum_{j}\left(N_{j, k} \times K\left(d_{i j}\right)\right)\right)\right\} .
\end{aligned}
$$




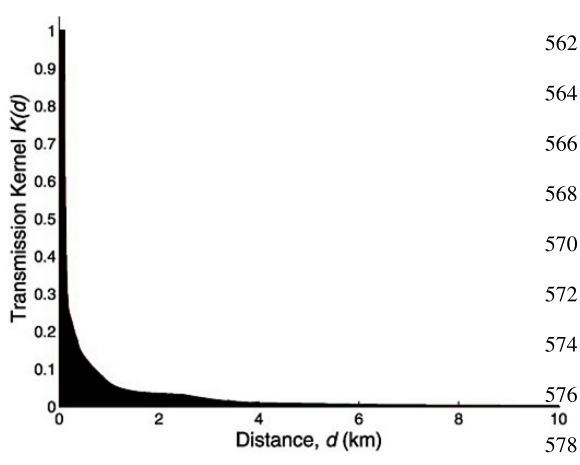

Figure 2. Transmission kernel: it was estimated by the Department of Environment, Food and Rural Affairs (DEFRA) ${ }^{2}$ and can be represented as a continuous function [17] or as a histogram [23]. It represents the multiplicative relative risk of transmission as a function of the distance from an IP.

In this model, we consider two animal species, cows and sheep, denoted by the subscript $k(k=1,2)$. The probability $P e_{i}$ that a susceptible farm $i$ is infected by an infectious farm $j$ first depends upon the number of cows and sheep in each farm (respectively $N_{i, k}, N_{j, k}$ ): the larger the herd the greater the risk of disease transmission. Second, the model depends on farm i's neighborhood. We consider a Transmission $\operatorname{Kernel}\left(K\left(d_{i j}\right)\right)$ that varies with the distance between farm $i$ and $j, d_{i j}$. The kernel represents the relative risk of transmission as a function of distance from an Infected Premise (IP) and is equal to one if farm $i$ was adjacent to an IP (Fig. 2). This kernel was previously estimated from the distribution of distances between identified infectious contacts up to $10 \mathrm{~km}$ by the Department of Environment, Food and Rural Affairs (DEFRA) in the UK. Third, the probability of transmission was different for the two species, through the parameters $S u_{k}$ and $T r_{k}$. $S u_{k}$ denotes the relative risk for a cow to catch the disease compared to a sheep - also known as the susceptibility parameter. $\operatorname{Tr}_{k}$ denotes the rate of disease spread - also known as the transmissibility parameter (Tab. I). These parameters were estimated by Keeling et al. [18] and validated on the UK 2001 epidemic (see Tab. I for their values). The probability for each farm to be infected by neighboring farms in a $10 \mathrm{~km}$ radius buffer [16] was estimated daily and the infection process from the susceptible state to the latent state was assessed by the Monte Carlo sampling method [24]. Next, each latent farm was individually tracked each day and stayed in each state according to the specific mean length of the transition state.

In France, we simulated 200 epidemics over a period of 365 days in order to ensure stable results, since the same patterns were observed by repeating several runs of 200 epidemics (about $6 \mathrm{~h}$ to be complete). The program was written in the $\mathrm{C}$ language and the results were analyzed with Matlab (v. 6.0). The model started with 50 initially infected farms; this number was in agreement with what was observed in the 2001 UK outbreak ${ }^{2}$. We randomly chose the 50 initial infected in regards to farm density and kept the same initial infected throughout the 200 simulation runs. We also performed several scenarios with different sets of 50 initial infected farms and chose to present the results of one "standard scenario". The results were represented by the mean and non-parametric $95 \%$ confidence limits (i.e. the 2.5 th and 97.5 th percentiles of the resulting distribution of the number of cases defined as the number of infected farms). We also provided the peak of the epidemic defined as the maximum of the daily mean incidence and the probability that an epidemic ended after one year by calculating the percentage of ended epidemics out of the 200 simulated epidemics. An epidemic was considered over when no cases were reported over 9 days (the maximum lag time before the disease is seen by the authorities).

\footnotetext{
2 Department of Environment, Food and Rural Affairs (DEFRA), foot-and-mouth disease http:// www.defra.gov.uk/footandmouth/ [consulted 14 February 2003].
} 
Table I. Values of the parameters $S u_{k}$ (Susceptibility parameter) and $\operatorname{Tr}_{k}$ (Transmissibility) for the two species, cows and sheep.

\begin{tabular}{|c|c|c|c|}
\hline Symbol & Species & Definition & Values (bounds) \\
\hline \multirow{2}{*}{$S u_{k}$} & Cow* & \multirow{2}{*}{ Susceptibility: risk of catching the disease } & $15.2(\mathbf{1 0}-\mathbf{2 0})$ \\
\hline & Sheep & & 1 \\
\hline \multirow[b]{2}{*}{$\operatorname{Tr}_{k}$} & Cow* & \multirow{2}{*}{ Transmissibility: rate of spreading the disease } & $4.3 .10^{-7}(\mathbf{2 . 3 6}-\mathbf{4 . 3})$ \\
\hline & Sheep* & & $2.36 .10^{-7}(\mathbf{2 . 3 6}-\mathbf{4 . 3})$ \\
\hline
\end{tabular}

* Indicates the parameters used in the multivariate sensitivity analysis.

\subsection{Control strategies}

The effect of control strategies such as pre-emptive culling and ring vaccination may be studied with this model. A farm, which fell within a selected buffer around an infected farm, could be classified as Contiguous Premises $(\mathrm{CP})$ and might be culled or vaccinated after a delay of two days ${ }^{2}$. This concerned Susceptible, Latent and Subclinical farms. The vaccine used was an emergency one whose efficacy is estimated to be $85 \%[3,19]$. The protection was conferred four days after injection and lasted for 4 to 6 months. The vaccine was considered to have no efficacy with animals belonging to Latent or Subclinical farms. In our model, the farms which were infected within the four days after vaccination, were classified as Latent [3, 4, 19, 25] and the farms for which vaccination was not successful were classified as Susceptible.

The standard control strategy was defined as the culling of animals from an IP within three days the first month of the epidemic, two days during the second month and one day after the third month ("standard control") ${ }^{2}$. This corresponded to what was observed in the 2001 UK outbreak. We explored four other types of control policies: (1) culling animals from an IP within $24 \mathrm{~h}$ for the duration of the epidemic ("policy control"), (2) pre-emptive culling within a $0.5\left(0.8 \mathrm{~km}^{2}\right), 1\left(3.1 \mathrm{~km}^{2}\right), 1.5\left(7.1 \mathrm{~km}^{2}\right)$ $\mathrm{km}$ radius buffer around an IP, (3) ring vaccination within a $3\left(28.3 \mathrm{~km}^{2}\right), 5\left(78.5 \mathrm{~km}^{2}\right)$, $10\left(314.2 \mathrm{~km}^{2}\right) \mathrm{km}$ radius buffer around an IP and 4) the combination of pre-emptive culling within a $0.5 \mathrm{~km}$ radius and ring vac- cination within a $3 \mathrm{~km}$ radius. All the farms within the radius buffer around an IP followed control strategies. We used the nonparametric Kruskall-Wallis test [7] to assess the significance of the various control strategies for the number of cases, the number of culled animals and the number of vaccinated animals.

\subsection{Farm network in France}

For reasons of confidentiality, we were unable to obtain the exact geographic coordinates of the French farms. We obtained data at the town scale from the Statistics Office of the Ministry of Agriculture and Fisheries (AGRESTE) ${ }^{3}$ providing for each of the 36604 French towns the number of animals, the number of holdings and the surface area. Among all these towns, 23544 owned farms, for a country wide total of approximately 280000 holdings with about 20 million cows and about 7 million sheep. The town was assumed to be a square whose boundaries are estimated from its surface area and from the geographical coordinates of its center. We distributed the location of the farms and the number of cows and sheep within each town in the following manner. First, within each town boundary, the location of the farm was uniformly distributed allowing spatial clustering of farms at the town level. Second, the number of animals

\footnotetext{
${ }^{3}$ Ministère de l'Agriculture et de la Pêche, Service central des enquêtes et études statistiques, Agricultural Census 2000 : la fiche comparative France métropolitaine (on CD-ROM).
} 
in each holding followed a normal distribution with the mean as the average number of sheep or cows in the town. The standard deviation followed the same trend as the mean (for farms with higher mean values the standard deviation was assumed to be greater) and was chosen so that the lower bound of the $95 \%$ confidence interval was greater than 0 . The number of animals in each holding was bounded between positive extreme values. This resulted in an empirical right-skewed distribution in agreement with statistics obtained at the town level ${ }^{3}$ and allowing for more variability than with other known right-skewed distributions. The number of mixed farms (i.e. farms with both sheep and cattle) was simulated according to the data obtained at the town level: in towns with both species a few mixed farms were simulated.

\subsection{High-risk zones}

The model provided a map of high-risk zones for the disease based on the basic reproduction number $R_{0}$ [1]. It was estimated empirically by simulating infection of an initial farm and then counting the number of secondary cases generated by this farm for the length of the infectious period (8 days at the onset of the epidemic). Once a farm was secondarily infected, its ability to transmit the disease was set to 0 . In order to build a map of $R_{0}$ values, we performed 100 simulations for each of the 280000 farms; it took about one month to be complete. Only the farms with mean $\mathrm{R}_{0}$ greater than 1 were represented as a colored square on the map, which allowed us to identify high-risk zones in France.

\subsection{Sensitivity analysis}

Two kinds of sensitivity analysis were carried out in order to evaluate, first, the influence of intrinsic disease parameters and second, spatial configurations on the annual cumulative number of cases.

First, since the susceptibility and transmissibility parameters were estimated from the UK epidemic, they might encompass other factors present in the UK epidemic, but not in France. In order to address this issue, we performed a multivariate sensitivity analysis to assess the uncertainties concerning three parameters that describe the disease transmission ( $T r$ for both species and $S u$ for cows, $S u$ for sheep is the reference and thus set to 1). In order to evaluate the impact of these parameters, we sampled 50 sets of the three disease parameters in uniform distributions (bounds described in Tab. I) thanks to a Latin Hypercube Sampling scheme [5]. We calculated the Partial Rank Correlation Coefficients (PRCC) between each parameter and the mean cumulative number of predicted cases.

Second, since we did not know the real distribution of the farms on the town surface area, we also performed a univariate sensitivity analysis in order to assess the impact of the global distribution of the farms. Two scenarios were explored; first, the farms were distributed all over the town surface area, second, the farms were only distributed in a quarter of the town surface area (the quarter was randomly chosen to be on the left, the right upper, the left lower or the right lower of the town). The initial infected were located in the same town throughout these two scenarios and we evaluated the impact on the mean number of cumulative cases.

\subsection{Validation}

In order to check that the model was in agreement with previous studies, we reproduced the UK epidemic with similar control strategies used in 2001: IP culled within 1 to 3 days, CP culled within 2 days, DC culled according to a ratio resulting from epidemiological surveys and extensive culling (within $3 \mathrm{~km}$ around an IP) in the most affected counties ${ }^{2}$. We then compared the temporal pattern of cases between the prediction of the model and the observed cases. This type of work was similar to what had already been done following the 2001 
UK epidemic $[9,19]$ thus we then mainly focused on the consequences in France.

UK data were collected from two sources provided by DEFRA ${ }^{2}$ : the Disease Control System (DCS) database which contained the confirmed infection by medical examination or serological test and slaughter date for the 2030 IP and the 2000 Census database which contained the number of animals (cows, sheep, goats, pigs and deer) for each of about 120000 farms and also the geographical coordinates and the size (ha) of about 80000 farms. After combining the information from these databases, we simulated epidemics within a network of about 56000 British farms located mainly in regions highly affected by the disease with approximately 4 million cows and 18 million sheep. According to the DCS database, all farms where the infection date was before the national movement restriction was applied were considered as initially infected. Thus 53 farms were classified as initially infected in the model implementation. The second database from the Census was used to estimate the Euclidean distance between the farms.

\section{RESULTS}

\subsection{Validation}

While simulating the epidemic in the UK according to the observed control measures, we found that the number of simulated cases would be 2050 (1 810; 2 300) and the epidemic peak would be 44 in one day, 36 days after the limitation of movements was put into place. In the 2001 UK epidemic, 2030 farms were infected with a peak of 51 cases at day 31 .

\subsection{Standard scenario}

The size of an epidemic, simulated in France from the same 50 initial infected farms with standard control policies would be 16350 infected farms, with a $95 \%$ con- fidence interval ( $8610 ; 20$ 860). Thus 5.9\% of the national susceptible farms would be infected; the epidemic peak would be 87 cases at day 122 (Fig. 3) and only $1 \%$ of the epidemic would be over after 365 days. The most affected counties would be BasseNormandie, Pays-de-la-Loire and Bretagne, which would account for $47.5 \%$ of the total simulated cases; the less affected cases would be Auvergne, the Midi-Pyrénées and the Limousin that would account for $21 \%$.

\subsection{Control policies}

The pre-emptive culling for a ring radius ranging from 0.5 to $1.5 \mathrm{~km}$ and ring vaccination from 3 to $10 \mathrm{~km}$ had the greatest impact on reducing the number of cases (decrease greater than 80\%) (Fig. 4A), animals culled (greater than 65\%) (Fig. 4B) and the length of the epidemic. Culling the animals within $1.5 \mathrm{~km}$ associated with a ring vaccination within $3 \mathrm{~km}$ would lead to 1110 cases $(610 ; 1590)$ with $38 \%$ of the simulated epidemics over at 6 months and $97.5 \%$ over at 1 year. For the pre-emptive culling, increasing the culling radius would decrease the total number of farms following slaughtering policies over the whole year (a $64 \%$ decrease from 0.5 to $1.5 \mathrm{~km}$ ) (Fig. 4B) but during the first days of applying control strategies the number of farms to be culled preemptively would be very high. There is a low impact in increasing the radius circle from 1 to $1.5 \mathrm{~km}$ concerning the relative decrease in the number of cases (Figs. 4A and 4B). Vaccinating animals within a $5 \mathrm{~km}$ radius circle decreases the number of infected farms; a lower number of farms would require vaccination than at a 3 or $10 \mathrm{~km}$ radius. At a $3 \mathrm{~km}$ radius the epidemic would last longer requiring more farms to be vaccinated. At a $10 \mathrm{~km}$ radius the number of simulated cases would be similar to the one at $5 \mathrm{~km}$ but the number of farms to be vaccinated at the onset of the epidemic would be higher. (Figs. 4A and 4C). All the differences presented here in the number of cases, number of animals 
A

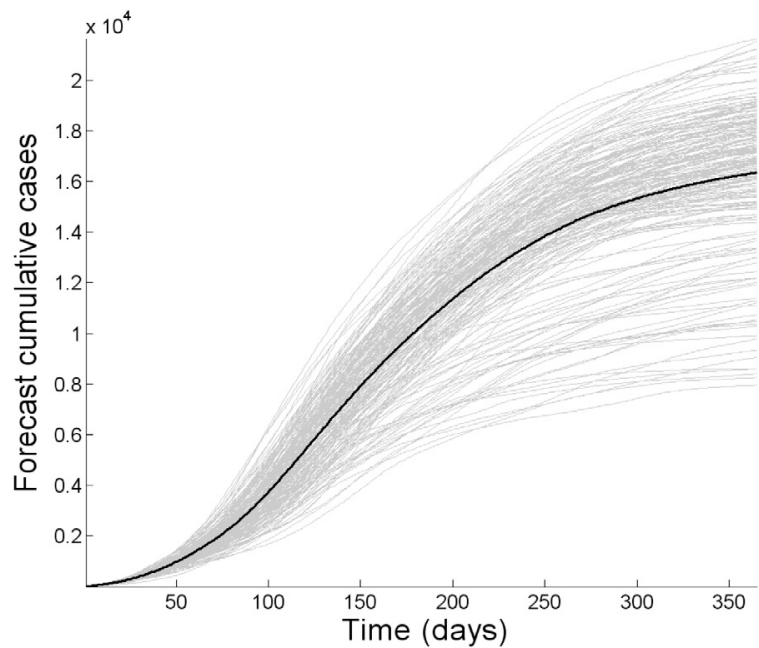

B

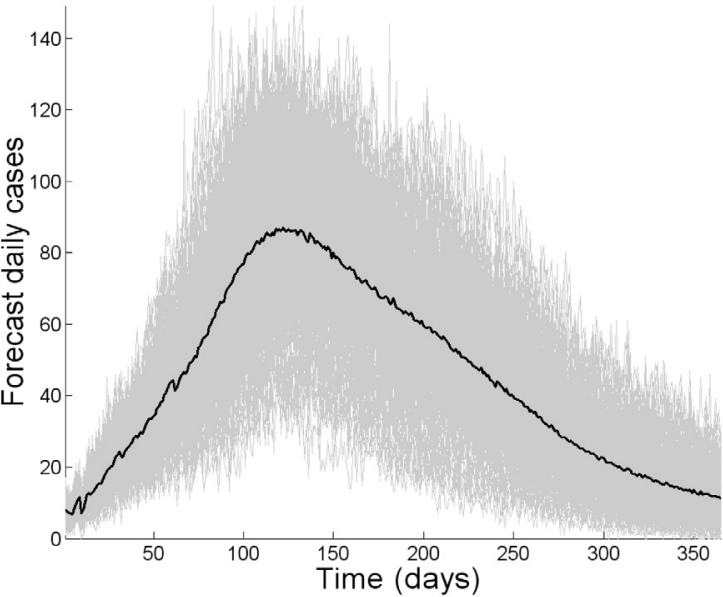

Figure 3. Forecast in France with the 50 initial infected farms from the reference scenario: (A) Forecast cumulative cases, (B) Forecast daily incidence. Black line represents the mean. The model results shown are from 200 simulations.

culled and number of vaccinated farms are significant $(p<0.001)$.

\subsection{High-risk zones}

Estimations of $\mathrm{R}_{0}$ allowed us to identify counties of high risk of FMD spreading if the virus was imported from a county of low risk. If the lower limit of the $95 \%$ non-par- ametric confidence interval for $\mathrm{R}_{0}$ was greater than 1 in a specific county, disease would spread. The risk of spread was likely to be important if the virus was imported into the southwest or northeast of the Paysde-la-Loire, south of the Limousin and of Bourgogne, east of the Midi-Pyrénées and Haute-Normandie and finally into the southwest of Aquitaine and northeast of Picardie 

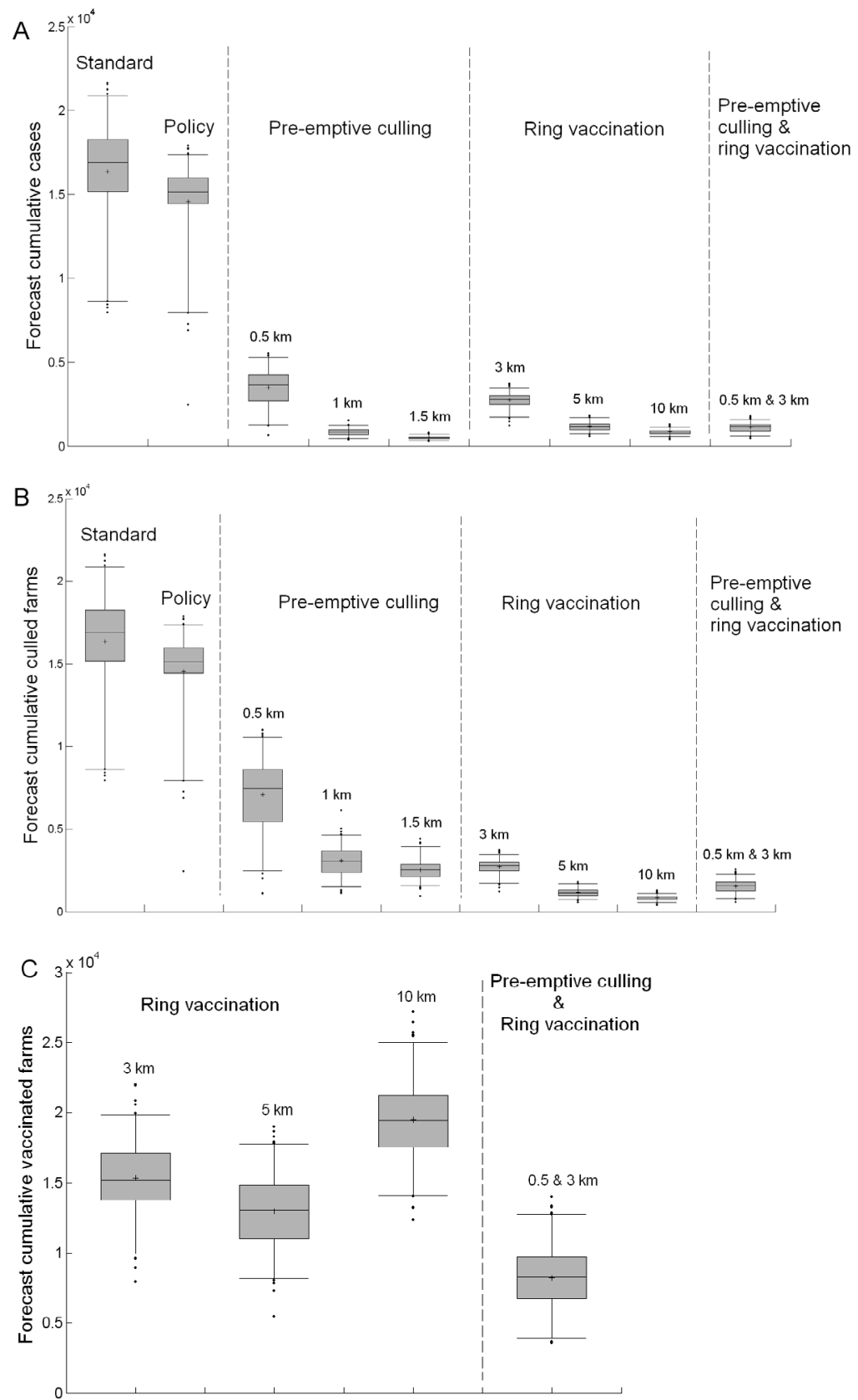

Figure 4. Simulation of a set of control measures with the 50 initial infected farms of the reference scenario: (A) Forecast cumulative cases, (B) Forecast culled farms, (C) Forecast vaccinated farms (Box plot main square represents the $25 \%$ and $75 \%$ quantiles, the mean is represented by the cross, the median by the black line and the dots represent extreme values over the 2.5 th or the 97.5 th percentiles). 


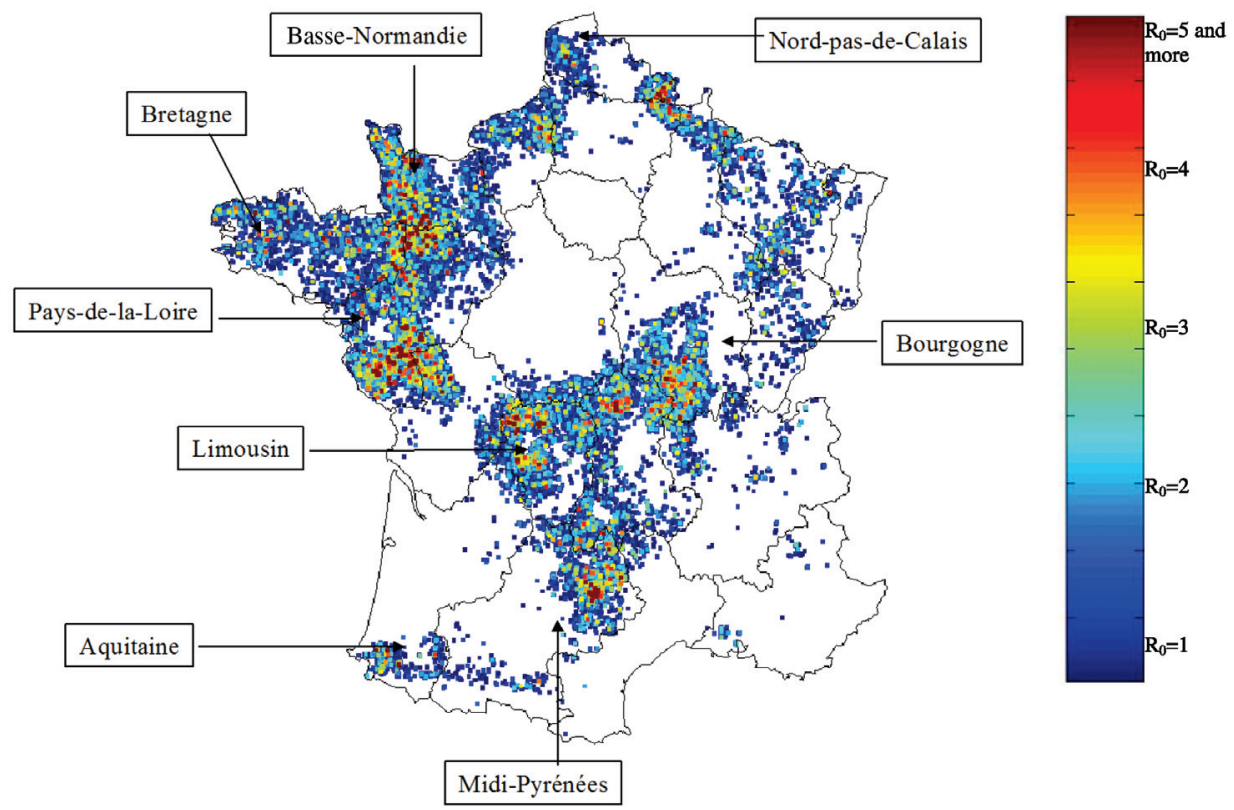

Figure 5. Map of farm $\mathrm{R}_{0}$ values on French territories (only farms that mean $\mathrm{R}_{0}$ over 100 simulations is greater than 1 are drawn).

(Fig. 5). In regions where color was red displayed, a clustering of high $\mathrm{R}_{0}$ and high values were due to a high density of farms and a high number of cows and sheep. Thus the maps emphasized the impact of initial infected farm location compared to the number of initially infected: increasing the number of initially infected in regions with low $\mathrm{R}_{0}$ values would unlikely lead to an epidemic.

\subsection{Sensitivity analysis}

The multivariate sensitivity analysis demonstrated the lower impact of the parameters related to sheep $(\mathrm{PRCC}=0.34)$ compared to cow parameters that had a similar impact $(\mathrm{Su}, \mathrm{PRCC}=0.88$ and $T r, \mathrm{PRCC}=$ 0.78).

When farms were located in a quarter of the area of the town rather than uniformly distributed, the peak would be earlier and greater and the total number of cumulative case would be larger $(20.9 \%$ of the initial susceptible farms and the peak is at day 88).

\section{DISCUSSION}

The results of this model provide decision-makers with information useful for epidemic response planning. We presented the results of one particular scenario without varying the location of the 50 initially infected farms to control variability. The epidemic dynamics of this scenario were close to what would have happened while varying the location of the 50 initially infected farms for each simulation run (data not shown). Mathematical modeling is a useful mean to assess the potential impact of epidemics; epidemiological surveys would help to identify potential long-range contacts and dangerous contacts. The model allows for the study of local dissemination of FMD in France due to direct (pasture) or indirect (people, vehicles) contact under 
different assumptions and it forecasts the consequences of virus introduction. Because this model is at the farm level, it allows for the creation of a map of high-risk areas in France for the spread of FMD once the virus has been imported. If the virus was imported in a high-risk area such as the Pays-de-laLoire, drastic and targeted control measures should be used such as pre-emptive culling or ring vaccination in a large perimeter. If the virus was imported in low risk zones such as Provences-Alpes-Côte-d'Azur, the control measures could be applied in a smaller perimeter.

The model demonstrated that culling animals only in the infected farms would not be sufficient to stop the spread of the disease, in agreement with other studies [27]. Culling or vaccinating animals from the farms around an infected farm would decrease the number of forecast cumulative cases and the length of the epidemic. However, pre-emptive culling becomes difficult to institute because of the strong and increasing opposition of veterinarians, herd owners and residents [6]. It may be difficult to apply culling policies to a large area because of the large number of farms located in the area of the pre-emptive culling. Ring vaccination, when vaccinated animals are not culled after the epidemic is over $^{1}$, preserves all animals, particularly animals with high genetic value, and prevents biological pollution. When animals are burned in open air, dioxin may be released in the atmosphere and when the animals are buried the pathogenic agent of bovine spongiform encephalopathy may be present in the soil ${ }^{4}$. Trivalent vaccines are used and are available. The number of doses and the strains are reviewed annually based on advice from the Institute of Animal Health at Pirbright (500 000 doses) and on strains which present the greatest risk. In addition to the countries' own stocks of

\footnotetext{
${ }^{4}$ Meikle J., Brown P., Maff knew of BSE risk before cattle burials [on line] (2001) http://www.guardian.co.uk/bse/article/0,2763,499626,00.html [consulted 17 July 2003].
}

FMD antigens, a wider range of strains is provided by the EU Vaccine Bank for emergency use (5 millions doses) ${ }^{2}$. The direct cost is estimated at around $€ 0.5$ to $€ 0.7$ per dose [20] and the indirect cost from $€ 1.2$ to $€ 2.4$ considering the scale of the vaccination program action and vaccine storage. Once the animals are vaccinated in a country, the country cannot export until six months after the last case. When no vaccination has been used in an epidemic, exportation bans end three months following the last case. The cost of one week without exportation is estimated to be around $€ 50$ million [21].

According to our model's results, when farms are tightly clustered (i.e. located in a quarter of the town surface area), the disease first spreads quickly within the quarter (or cluster; intra-cluster transmission). Thus the number of farms simultaneously affected is large. Therefore even if a susceptible cluster is far from the infected one, if all the farms are infected the same day, the probability to be infected would be larger and would counterbalance the distance effect (inter-cluster transmission). If the number of farms within the cluster is low, intercluster transmission would be unlikely to occur since the infectious potential of this cluster would be low. When there is no cluster of farms, the disease is transmitted from farm to farm and not from cluster to cluster and so the spread may be slower.

We applied a model similar to a previous one conducted by Keeling et al. and validated on the UK 2001 epidemic data; our model results simulated in the UK were clearly in line with the observed and other previous mathematical works [18]. Therefore we used the same parameters, susceptibility and transmissibility, as the ones estimated by Keeling et al. [18] during the 2001 epidemic. These parameters may encompass other information such as climatic conditions or the efficacy of veterinary services. Nevertheless they were in agreement with the biological process since cows are the most susceptible species and cows and 
sheep excrete similar levels of virus. Since they describe disease transmission and since breeding activity is similar in France and the UK, they should be generic enough to be applied in other countries. The last major epidemic was in 1981 and at this time the cattle were vaccinated but no account of which farm were vaccinated and where it was located was kept. Thus no validation of the model was possible using French data and the only way to explore the consequences of FMD was to use parameters estimated from other epidemics, as it done previously with the epidemic from 1967-1968 [11] and more recently in 2001 [9, 10, 18, 19, 22, 23]. Furthermore, Keeling et al. [19] argue that the epidemic trend was not sensitive to the precise shape of the infection Kernel.

The model is probably not well adapted to outbreaks occurring in countries with extensive animal breeding. With extensive breeding, the hypothesis assuming that when one animal on a farm is infected by the disease all the animals are infected may not be reasonable. Contacts between animals are very close in intensive breeding and so "within farm" behavior of the disease is likely to have a lower impact on the spread of the disease than the between farm behavior. Nevertheless the model is applicable in France where extensive breeding mainly occurs in a few areas where the $\mathrm{R}_{0}$ values are low (e.g. Provence-Alpes-Côte d'Azur). Finally, the role of pigs was not taken into account, leading us to interpret with caution the results from regions with high pig density, such as Brittany. We did not include the role of pigs in the epidemic for two main reasons. First, no reliable estimate of the transmissibility and susceptibility parameters are so far available with this type of model. If an epidemic occurs in France and if pigs holding are infected, such parameters ( $\mathrm{Su}$ and $\mathrm{Tr}$ for pigs) could be estimated from the data and the species could be easily added to the model. Second, the swine population and farming is aggregated and highly regulated in a few regions in France. This type of farming is isolated from other types and the level of safety and hygiene is very high. Therefore these herds are under surveillance and pigs are the first species to be slaughtered in case of an epidemic.

In order to assess the dissemination of the virus from the date of importation to the date when the disease is recognized, we could use either data from commercial movements of animals or from zones of high breeding activities. The main difficulty with commercial movements is the accumulation of data and then summarizing this information at a regional level would be a poor approximation. Taking a sample of movement is an important idea but mainly applicable when we study exchange at a country level, i.e. exportation of cattle within the European Community or between continents. Within a country and more particularly at a farm level, the contacts correspond to local contacts based on commercial, human movement or on material. Thus the scale of contact is large and unfortunately can not be studied in a relevant way on a national scale. Adding this information into a model of the type presented here adds many uncertainties and computational intractability. This would require other mathematical tools as deterministic models based on contact matrix for computational reasons and it would be part of future research. Thus, considering the lack of precise data, the number of initially infected farms was simulated in regards to farm density. High farm densities are assumed to be at a higher risk of virus importation and are correlated with greater breeding activities. In 2001 in the UK the virus was disseminated through markets located in high sheep and cattle density regions [9].

The reintroduction of FMD in France would have a high burden on animal health. We have shown that adapting the Keeling model provides a tool to explore the epidemic's dynamics and the impact of control strategies. These simulations help to identify which strategies (culling or vaccination) would be better to stop the transmission of the disease depending on the 
administrative region affected. Since 50 initial infected farms located in low-risk areas would probably not lead to an epidemic, the impact of the initial infected farm location underlines the need of quickly reporting the initial cases. The impact of farm clusters in the epidemic course highlights the fact that using a spatial and individual model requires an accurate positioning of herds. Thus it would be useful to develop a GIS to provide the distribution of farms in France and to aid in developing effective control strategies.

\section{ACKNOWLEDGEMENTS}

We wish to thank B. Durand from AFSSA (Agence Française pour la Sécurité Sanitaire des Aliments), Epidemiology Unit, for his comments and corrections on the manuscript.

\section{REFERENCES}

[1] Anderson R.M., May R.M., Infectious diseases of humans: dynamics and control, Oxford University Press, Oxford, 1991.

[2] Bailey N.T.J., The mathematical theory of infectious diseases and its applications, Griffin, London, 1975.

[3] Bates T.W., Thurmond M.C., Carpenter T.E., Description of an epidemic simulation model for use in evaluating strategies to control an outbreak of foot-and-mouth disease, Am. J. Vet. Res. 64 (2003) 195-204.

[4] Bates T.W., Thurmond M.C., Carpenter T.E., Results of epidemic simulation modeling to evaluate strategies to control an outbreak of foot-and-mouth disease, Am. J. Vet. Res. 64 (2003) 205-210.

[5] Blower S.M., Dowlatabadi H., Sensitivity and uncertainty analysis of complex models of disease transmission: an HIV model, as an example, Int. Stat. Rev. 62 (1994) 229-243.

[6] Bouma A., Elbers A.R., Dekker A., de Koeijer A., Bartels C., Vellema P., van der Wal P., van Rooij E.M., Pluimers F.H., de Jong M.C., The foot-and-mouth disease epidemic in The Netherlands in 2001, Prev. Vet. Med. 57 (2003) 155-166.

[7] Conover W.J., Practical nonparametric statistics, John Wiley \& Sons, New York, 1980.
[8] Durand B., Mahul O., An extended state-transition model for foot-and-mouth disease epidemics in France, Prev. Vet. Med. 47 (1999) 121-139.

[9] Ferguson N.M., Donnelly C.A., Anderson R.M., The foot-and-mouth epidemic in Great Britain: pattern of spread and impact of interventions, Science 292 (2001) 1155-1160.

[10] Ferguson N.M., Donnelly C.A., Anderson R.M., Transmission intensity and impact of control policies on the foot-and-mouth epidemic in Great Britain, Nature 413 (2001) 542-548.

[11] Gerbier G., Bacro J.N., Pouillot R., Durand B., Moutou F., Chadoeuf J., A point pattern model of the spread of foot-and-mouth disease, Prev. Vet. Med. 56 (2002) 33-49.

[12] Gibbens J.C., Sharpe C.E., Wilesmith J.W., Mansley L.M., Michalopoulou E., Ryan J.B., Hudson M., Descriptive epidemiology of the 2001 foot-and-mouth disease epidemic in Great Britain: the first five months, Vet. Rec. 149 (2001) 729-743.

[13] Gudelj I., White K.A., Spatial heterogeneity, social structure and disease dynamics of animal populations, Theor. Popul. Biol. 66 (2004) 139-149.

[14] Haydon D.T., Woolhouse M.E., Kitching R.P., An analysis of foot-and-mouth-disease epidemics in the UK, IMA J. Math. Appl. Med. Biol. 14 (1997) 1-9.

[15] Hugh-Jones M.E., Tinline R.R., Studies on the 1967-1968 foot-and-mouth disease epidemic: incubation period and herd serial interval, J. Hyg. 77 (1976) 141-153.

[16] Kao R.R., Landscape fragmentation and footand-mouth disease transmission, Vet. Rec. 148 (2001) 746-747.

[17] Kao R.R., The role of mathematical modelling in the control of the 2001 FMD epidemic in the UK, Trends Microbiol. 10 (2002) 279-286.

[18] Keeling M.J., Woolhouse M.E., Shaw D.J., Matthews L., Chase-Topping M., Haydon D.T., Cornell S.J., Kappey J., Wilesmith J., Grenfell B.T., Dynamics of the 2001 UK footand-mouth epidemic: stochastic dispersal in a heterogeneous landscape, Science 294 (2001) 813-817.

[19] Keeling M.J., Woolhouse M.E., May R.M., Davies G., Grenfell B.T., Modelling vaccination strategies against foot-and-mouth disease, Nature 421 (2003) 136-142.

[20] Leforban Y., How predictable were the outbreaks of foot-and-mouth disease in Europe in 2001 and is vaccination the answer? Rev. Sci. Tech. 21 (2002) 549-556. 
[21] Mahul O., Durand B., Simulated economic consequences of foot-and-mouth disease epidemics and their public control in France, Prev. Vet. Med. 47 (1999) 23-38.

[22] Matthews L., Haydon D.T., Shaw D.J., ChaseTopping M.E., Keeling M.J., Woolhouse M.E., Neighbourhood control policies and the spread of infectious diseases, Proc. R. Soc. Lond. B Biol. Sci. 270 (2003) 1659-1666.

[23] Morris R.S., Wilesmith J.W., Stern M.W., Sanson R.L., Stevenson M.A., Predictive spatial modelling of alternative control strategies for the foot-and-mouth disease epidemic in Great Britain, 2001, Vet. Rec. 49 (2001) 137144.

[24] Raeside D.E., Monte Carlo principles and applications, Phys. Med. Biol. 21 (1976) 181197.

[25] Saiz M., Nunez J.I., Jimenez-Clavero M.A., Baranowski E., Sobrino F., Foot-and-mouth disease virus: biology and prospects for disease control, Microbes Infect. 4 (2002) 11831192

[26] Sobrino F., Saiz M., Jimenez-Clavero M.A., Nunez J.I., Rosas M.F., Baranowski E., Ley V., Foot-and-mouth disease virus: a long known virus, but a current threat, Vet. Res. 32 (2001) 1-30.

[27] Tomassen F.H., de Koeijer A., Mourits M.C., Dekker A., Bouma A., Huirne R.B., A decision-tree to optimise control measures during the early stage of a foot-and-mouth disease epidemic, Prev. Vet. Med. 54 (2002) 301324.

[28] Yu P., Habtemariam T., Wilson S., Oryang D. Nganwa D., Obasa M., Robnett V., A riskassessment model for foot-and-mouth disease (FMD) virus introduction through deboned beef importation, Prev. Vet. Med. 30 (1997) 49-59. 